Madras Dominika, Rocka Agata, Piędel Faustyna, Psiuk Dominika, Nowak Emilia. Increase in IL-15 levels in patients with alopecia areata. Journal of Education, Health and Sport. 2020;10(9):76-83. eISSN 2391-8306. DOI http://dx.doi.org/10.12775/JEHS.2020.10.09.008 https://apcz.umk.pl/czasopisma/index.php/JEHS/article/view/JEHS.2020.10.09.008 https://zenodo.org/record/4014346

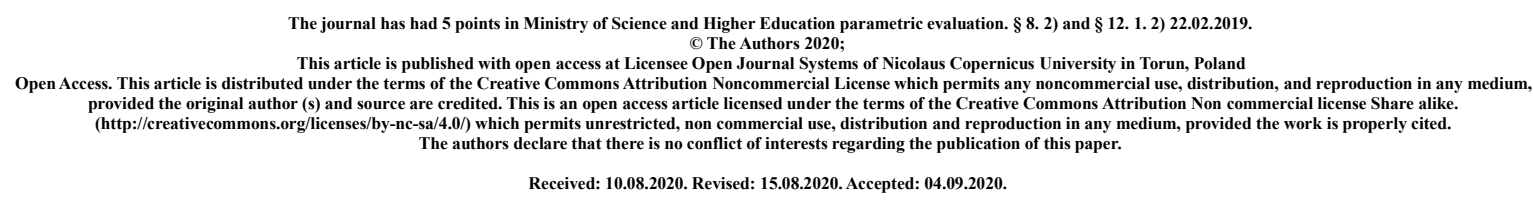

Increase in IL-15 levels in patients with alopecia areata

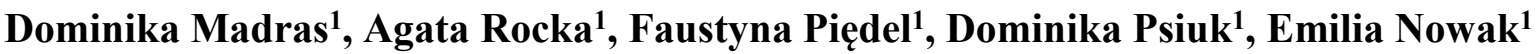 \\ ${ }^{1}$ Faculty of Medicine, Medical University of Lublin, Chodźki Street 19, 20-093 Lublin, \\ Poland
}

Dominika Madras; dkmadras@gmail.com; ORCID: 0000-0002-1777-4403

Agata Rocka; agatarocka2@gmail.com; ORCID:0000-0003-4738-3160

Faustyna Piędel; faustyna.piedel@gmail.com; ORCID:0000-0002-8280-498X

Dominika Psiuk; dominika.psiuk@gmail.com; ORCID:0000-0003-3319-3489

Emilia Nowak; emilia.m.nowak@wp.pl; ORCID:0000-0003-4012-2419

\title{
SUMMARY
}

Introduction and purpose: Alopecia areata (AA) is a condition that causes non-scarring hair loss (often with acute onset). Alopecia areata in the population occurs in $0.1-0.2 \%$ of people, with a similar frequency in men and women. Alopecia areata is an example of an autoimmune disease of the hair follicle. Hair loss in alopecia areata is caused by lymphocytic infiltration around the hair follicle and IFN- $\gamma$. IgG antibodies against hair follicle cells are also found in people suffering from alopecia areata. Recent studies have shown a significant increase in IL-15 in AA. The aim of the study was to review the current knowledge on the use of IL-15 in the treatment of alopecia areata.

A brief description of the state of knowledge: Interleukin-15 (IL-15) is a pleiotropic cytokine that exhibits multidirectional biological effects on various cell types. It affects the functions of the immune system, both innate and acquired, and therefore plays an important role in inflammation and during the immune response to infections and infestations. 
In the AA mouse model, antibody-mediated blockade of IFN- $\gamma$, interleukin-2 (IL-2), or interleukin-15 receptor $\beta$ (IL-15R $\beta$ ) prevented disease progression by minimizing the accumulation of CD8 $(+)$ NKG2D $(+)$ T cells in the skin and reducing the cutaneous IFN response. The concentration of IL-15 in patients with alopecia areata was significantly higher than in the control group. Moreover, the concentration of IL-15 increased in direct proportion to the area of alopecia, the highest value in patients with total alopecia.

For this reason, it is important to search for new medical treatments that will enable patients to stay physically healthy, and what is equally important, to remain mental health.

Conclusions: In addition, studies have shown an increase in IL-15 levels in patients with alopecia areata, which correlated with the duration of the disease. However, too few studies conducted so far do not allow conclusions to be drawn regarding the use of IL-15 as a therapeutic point.

Key words: alopecia areata, Il-15, immunology

\section{State of knowledge}

\section{Alopecia Areata}

Alopecia areata (AA) is a condition that causes non-scarring hair loss (often with acute onset). It can occur in any hair-bearing area of the skin (from small patches to extensive involvement), but the most visible areas are the scalp, chin area and eyebrows. Now, it is known that the condition is caused by an autoimmune disease of the hair follicles with a genetic background[1]. Alopecia areata affects both children and adults, regardless of hair color[2]. So far, there is no causal therapy for AA. Also, there is a lack of reliable long-term controlled trials assessing the treatment of alopecia areata and its impact on quality of life[3]. Alopecia areata is one of the most common autoimmune diseases. The pathogenesis is not fully understood. The lack of effective therapies leads to a constant search for new treatments[4].

\section{Epidemiology}

Alopecia areata in the population occurs in $0.1-0.2 \%$ of people, with a similar frequency in men and women. In $70-80 \%$, symptoms appear before the age of 40 . However, $48 \%$ of people may develop symptoms in the first and second decades of their lives, which makes alopecia areata the most common cause of hair loss in healthy children[5,6].

\section{Clinical presentation}

Most often, AA patches are single or multiple. The shape of the lesions is usually round or oval, it can also present the form of a wave at a circumference of the head (ophiasis), in some cases it can lead to complete hair loss (alopecia totalis). The skin in these areas remains unchanged, and hair follicles are intact. Nail plate was also noted in AA patients, more frequently in children $>40 \%$. The changes are manifested by nail pitting or making it lustreless and rough. The lesions may take various forms: trachyonychia, punctate leukonychia, spotted or red lunulae, transverse nail grooving (Beau's lines) or the rare separation of the nail plate from the matrix with nail loss (onychomadesis)[7]. 


\section{Autoimmune pathogenesis}

Alopecia areata is an example of an autoimmune disease of the hair follicle with a genetic background. Hair loss in alopecia areata is caused by lymphocytic infiltration around the hair follicle and IFN- $\gamma$. IgG antibodies against hair follicle cells are also found in people suffering from alopecia areata. Autoreactive $\mathrm{CD} 8+, \mathrm{CD} 4+, \mathrm{NK}$ and $\mathrm{pDC}$ cells infiltrate around the hair follicle in the growth phase (anagen). Increased activity of cytokines disrupts normal hair growth and terminates the anagen phase. Furthermore, the disease coexists with other autoimmune diseases and may be secondary to infection or inflammation[8].

\section{Alopecia areata and mental well-being}

Alopecia areata quickly leads to the loss of more and more hair. The resulting distortions can cause anxiety in patients and increase the risk of developing psychiatric problems[9].

Hair diseases are a serious problem for patients' lives, causing significant emotional and psychosocial stress. Study of comparison between alopecia areata (AA) and androgenetic alopecia (AGA) was conducted in the context of psychological changes in those groups of patients.The study included patients of the same age, gender, depression and comorbidities. The results showed that patients with AA had a worse quality of life compared to patients with AGA (mean dermatological quality of life index: 5.8 vs. 2.5). The study confirmed that patients with hair disease are more anxious, depressed and have a lower quality of life compared to the control group[10].

It seems unlikely that anxiety and depression play a major role in the etiopathogenesis of AA, but stressful life events can trigger the onset and/or exacerbation of the disease. In addition, AA appears to have a partially negative impact on health-related quality of life[11].

Many studies emphasize the role of psychological factors in the progression of the disease such as alopecia areata. Social exclusion, family problems are more common and have a greater impact on the daily life of AA patients than in non-AA patients [12], unfortunately, over time most of them develop psychological problems such as depression, anxiety and paranoid disorders[13,14,15,16,17].

Studies have also shown that the poor quality of life of these patients is significantly associated with depression[18]. It seems that patients with alopecia areata are mainly depressed, worried and hysterical, have a higher tendency to hypochondria and often experience conflicts in everyday contacts with other people [19]. In these patients, the suicide tendency is high [20]. Studies have shown that there is a significant relationship between hair loss and stress, the intensity of stress and stressful events [21]. There is evidence that hypnotherapy is effective in treating alopecia areata[22,23].

The evaluation of facial images of people with AA showed signs of depression, anxiety and neurosis $[24,25]$. The most important variable leading to mental illness in skin disorders is visible deformity[26]. The constantly exposed area (scalp) and the nature of the lesion (size and location of patch) significantly affect the mental condition of patients[27].

For this reason, it is important to search for new medical treatments that will enable patients to stay physically healthy, and what is equally important, to remain mental health. 


\section{Treatment}

Currently, numerous topical systemic agents are used to treat AA. However, there is no data assessing their efficacy, tolerability and safety.

Topical treatment includes glucocorticosteroids in the form of ointments, gels, lotions, foams or intradermally. Local therapy may be used in cases of limited spectrum disease. Possible indications for systemic treatment include rapid hair loss, extensive disease $(\geq 50 \%$ hair loss), chronic disease, severe stress, or a combination of factors. Systemic therapy includes glucocorticosteroids, methotrexate, cyclosporine, azathioprine, dapsone, mycophenolate mofetil, tacrolimus and sulfasalazine. Other treatment methods include: photochemotherapy (PUVA or PUVA-turban) and narrowband UVB phototherapy[28-30].

\section{IL-15}

Interleukin-15 (IL-15) is a pleiotropic cytokine that exhibits multidirectional biological effects on various cell types. It affects the functions of the immune system, both innate and acquired, and therefore plays an important role in inflammation and during the immune response to infections and infestations[31].

Blocking the IL-15 beta receptor (IL-15R $\beta$ ) caused a reduction in the number of $\mathrm{NKG} 2 \mathrm{D}+\mathrm{CD} 8+\mathrm{T}$ cells in the skin, which prevents the development of AA using an animal model. It was observed that the elevation of IL-15 and its IL-15R $\alpha$ receptor subunit in AA hair follicles. IL-15 acts as a pro-inflammatory cytokine and stimulates autoreactive $\mathrm{T}$ cells[32]. IL-15 acts through the Janus (JAK) -1, Jak3 pathway and uses the transcription activator STAT-5[33].

Both IFN- $\gamma$ and IL-15 are promising therapeutic targets in the treatment of alopecia areata[34].

In the AA mouse model, antibody-mediated blockade of IFN- $\gamma$, interleukin-2 (IL-2), or interleukin-15 receptor $\beta$ (IL-15R $\beta$ ) prevented disease progression by minimizing the accumulation of CD8 $(+)$ NKG2D (+) T cells in the skin and reducing the cutaneous IFN response. Systemically administered pharmacological inhibitors of Janus kinase (JAK) family protein tyrosine kinases (effectors of IFN- $\gamma$ cytokine receptors), prevented the development of AA, while topical administration promoted hair regrowth[32].

Duculan et al. performed a biopsy of the altered and unchanged scalp of 6 patients with AA before treatment and after topical methylprednisolone therapy to evaluate the activity of AA biomarkers. The qRT-PCR test before the treatment application showed a significant increase in the expression of inflammatory markers in the unchanged skin, in comparison with the non-affected skin, including e.g. IL-15 and JAK-3, and IL-2, IL-2RA as well as Th1, Th2, IL-12 / IL-23p40 and IL-32. In contrast, immunohistochemistry showed an increase in CD3 (+), CD8 (+) T cells, CD11c (+) dendritic cells and CD1a (+) Langerhans cells around the hair follicles of the scalp with lesions that decreased after treatment[35].

Ebrahim AA et al. study involved 80 people, including 40 patients with alopecia areata and 40 healthy people. The participants were examined in order to assess the condition of the scalp - locatization, number and size of alopecia patches, as well as the severity of the disease using the SALT scale. Then, the concentration of IL-15 was measured in all participants by the ELISA method. The concentration of IL-15 in patients with alopecia areata was significantly higher than in the control group $(33.98 \mathrm{pg} / \mathrm{ml}$ and $7.88 \mathrm{pg} / \mathrm{ml}$, respectively). 
Moreover, the concentration of IL-15 increased in direct proportion to the area of alopecia, the highest value in patients with total alopecia $(50.08 \mathrm{pg} / \mathrm{ml})$. There was also a relationship between the SALT score and IL-15 concentration - the higher the percentage of hair loss, the higher the IL-15 concentration observed[36].

Another study by Tabara K et al. included 42 patients (children) with alopecia areata, and 37 healthy children. Peripheral venous blood samples were collected from all participants to measure the concentration of the following cytokines - IL-6, IL-15, IL-17A and IFNgamma using the ELISA method. There was a significant increase in the concentration of all of the tested cytokines in the test group compared to the control group. Moreover, the concentration of IL-15 increased in direct proportion to the length of the disease, while the concentration of IL-17A decreased with the over two years long episode of disease and in the presence of thyroiditis. The highest mean concentration in the group of patients with alopecia areata among the tested cytokines was IFN-gamma $(158.70 \mathrm{pg} / \mathrm{ml})$, while IL-15 was in second place $(89.59 \mathrm{pg} / \mathrm{ml})[37]$.

The Bansal et al. study included 30 patients with alopecia areata with varying degrees of hair loss assessed by the SALT (Severity of Alopecia Tool Score). From each patient, a biopsy sample was taken from the margin of the lesion for histopathological and immunological examination, using CD4, CD8 antibodies and Tyr701 protein. The aim of the study was to measure the number of dermal papillae of hair follicles expressing Tyr701 and the number of CD4 + and CD9 + T cells in the periocular and intraocular regions[38].

In Kim et al. study the effect of ruxolitinib on human dermal papilla cells was observed. For this purpose, an in vitro model of previously interferon gamma-exposed cells was used, and the expression of the wnt/beta catenin pathway, JAK-STAT pathway and growth factors was examined using rt-PCR and Western Blot. Ruxolitinib did not affect cell viability, and activated some components of the wnt-beta catenin pathway, inhibited DKK1 expression, and also reversed IFN-dependent expression of a number of molecules, such as caspase-1, IL-1beta, IL-15 and IL-18, as a result reducing their expression. The drug increased the expression of some growth factors, and also inhibited the phosphorylation of JAK1, JAK2, JAK3, STAT1 and STAT3. Study results suggest that ruxolitinib therapy may prevent interferon gamma-induced hair loss[39].

JAK inhibitors mediate IL-15 signal transduction[40]. This may prevent the development of alopecia areata. JAK inhibitors represent a promising therapeutic option for people with alopecia areata. However, none of them (Tofacinib, Ruxolinib, Baricitinib) have been approved by the FDA for this indication, so far.

The efficacy and safety of Ruxolitinib and Tofacitinib were compared in 75 patients with severe alopecia areata. Patients were randomized to two groups and took JAK inhibitors for 6 months. Both people taking Ruxolitinib and Tofacitinib experienced hair regrowth. The change in SALT was $93.8 \pm 3.25$ in the Ruxolitinib group and $95.2 \pm 2.69$ in the Tofacitinib group. Despite the recurrence of alopecia in $2 / 3$ of the patients, both drugs can be described as promising in the treatment of alopecia areata[41]. 


\section{Conclusions}

Alopecia areata is a common disease. Often patients with AA struggle with mental problems (depression, anxiety). AA interferes with their proper functioning in society. The lack of effective treatment exacerbates the problem.

The pathomechanism of the disease is autoimmune. The ongoing research focuses primarily on assessing the impact of cytokine disturbances on the pathogenesis of AA. Scientists are conducting research on IL-6, IL-15, IL-17A and IFN-gamma. In addition, studies have shown an increase in IL-15 levels in patients with alopecia areata, which correlated with the duration of the disease. However, too few studies conducted so far do not allow conclusions to be drawn regarding the use of IL-15 as a therapeutic point.

\section{List of references:}

[1]Martinez-Mir A, Zlotogorski A, Gordon D, Petukhova L, Mo J, Gilliam TC, Londono D, Haynes C, Ott J, Hordinsky M, Nanova K, Norris D, Price V, Duvic M, Christiano AM (2007) Genomewide scan for linkage reveals evidence of several susceptibility loci for alopecia areata. Am J Hum Genet 80:316-328.

[2]Finner am. alopecia areata: clinical presentation, diagnosis, and unusual cases. Dermatol ther 2011;24:348-54.

[3]Slowinska m, Kardynal a, Warszawik o, Czuwara J, rudnickal: alopecia areata developing paralell to improvement of psoriasis during ustekinumab therapy. J Dermatol Case rep 2010,4: 15-17.

[4]Gilhar A, Etzioni A, Paus R. Alopecia areata. N Engl J Med. 2012;366(16):1515-1525.

[5]Safavi KH, Muller SA, Suman VJ, Moshell AN, Melton LJ III. (1995) Częstość występowania łysienia plackowatego w Olmsted County, Minnesota, 1975 do 1989. Mayo Clin Proc 70 (7): 628-633.

[6]Kyriakis KP, Paltatzidou K, Kosma E, Sofouri E, Tadros A, Rachioti E (2009) Alopecia areata prevalence by gender and age. J Eur Acad Dermatol Venereol 23 (5): 572-573

[7]Trüeb, R.M., Dias, M.F.R.G. Alopecia Areata: a Comprehensive Review of Pathogenesis and Management. Clinic Rev Allerg Immunol 54, 68-87 (2018).

[8]Simakou T, Butcher JP, Reid S, Henriquez FL. Alopecia areata: A multifactorial autoimmune condition. J Autoimmun. 2019;98:74-85.

[9]Pratt CH, King LE Jr, Messenger AG, Christiano AM, Sundberg JP. Alopecia areata. Nat Rev Dis Primers. 2017;3:17011.

[10]Titeca G, Goudetsidis L, Francq B, et al. 'The psychosocial burden of alopecia areata and androgenetica': a cross-sectional multicentre study among dermatological out-patients in 13 European countries [published correction appears in J Eur Acad Dermatol Venereol. 2020 May;34(5):1119]. J Eur Acad Dermatol Venereol. 2020;34(2):406-411.

[11]Güleç AT, Tanriverdi N, Dürü C, Saray Y, Akçali C. The role of psychological factors in alopecia areata and the impact of the disease on the quality of life. Int $\mathrm{J}$ Dermatol. 2004;43(5):352-356.

[12]Alopecia areata: psychiatric comorbidity and adjustment to illness.

Ruiz-Doblado S, Carrizosa A, García-Hernández MJ Int J Dermatol. 2003 Jun; 42(6):434-7.

[13]Ruiz-Doblado S, Carrizosa A, García-Hernández MJ. Alopecia areata: psychiatric comorbidity and adjustment to illness. International Journal of Dermatology. 2003;42(6):434-437. 
[14]Grahovac T, Ružić K, Šepić-Grahovac D, Dadić-Hero E, Pavešić Radonja A. Depressive disorder and alopecia. Psychiatria Danubina. 2010;22(2):293-295.

[15]Ataseven A, Saral Y, Godekmerdan A. Serum cytokine levels and anxiety and depression rates in patients with Alopecia areata. Eurasian Journal of Medicine. 2011;43(2):99-102.

[16]Koblenzer CS. Psychocutaneous Disease. Orlando, Fla, USA: Grune \& Stratton; 1987. [17]Arnold LM. Psychogenic excoriation: clinical features, proposed diagnostic criteria, epidemiology and approaches to treatment. CNS Drugs. 2001;15(5):351-359. [18]Associations between skin diseases and quality of life: a comparison of psoriasis, vitiligo, and alopecia areata. Ghajarzadeh M, Ghiasi M, Kheirkhah S Acta Med Iran. 2012; 50(7):5115 .

[19]Psychological status of patients with alopecia areata. Alfani S, Antinone V, Mozzetta A, Di Pietro C, Mazzanti C, Stella P, Raskovich D, Abeni D Acta Derm Venereol. 2012 May; 92(3):304-6.

[20]Layegh P, Arshadi H, Shahriari S, Pezeshkpour F, Nahidi Y. A comparative study on the prevalence of depression and suicidal ideation in dermatology patients suffering from Psoriasis, Acne, Alopecia areata and Vitiligo. Iranian Journal of Dermatology. 2010;13:106111.

[21]Arbabi N, Salami F, Forouzesh F, Gharehbeglou M, Riyahin A, Shahrzad M. Effects of stress and stressful events on Alopecia areata. Life Science Journal. 2013;10(6)

[22]Hypnosis and alopecia areata: Long-term beneficial effects on psychological well-being. Willemsen R, Haentjens P, Roseeuw D, Vanderlinden J Acta Derm Venereol. 2011 Jan; 91(1):35-9.

[23]Hypnotic approaches for alopecia areata. Willemsen $\mathrm{R}$, Vanderlinden $\mathrm{J}$ Int $\mathrm{J}$ Clin Exp Hypn. 2008 Jul; 56(3):318-33.

[24]Aghaei S, Saki N, Daneshmand E, Kardeh B. Prevalence of psychological disorders in patients with alopecia areata in comparison with normal subjects. ISRN Dermatol. 2014 Mar 9;2014:304370.

[25]Holter RF, Bargoon C. Psychological consideration of the skin in childhood. Pediatric Clinics of North America. 1961;8:719-739.

[26]Holter RF, Bargoon C. Pediatric Clinics of North America. 1961;8:719-739.

[27]Dermatopsychosomatics: classification, physiology, and therapeutic approaches. Medansky RS, Handler RM J Am Acad Dermatol. 1981 Aug; 5(2):125-36.

[28]Cranwell WC, Lai VW, Photiou L, et al. Treatment of alopecia areata: An Australian expert consensus statement. Australas J Dermatol. 2019;60(2):163-170.

[29]França K, Castillo D, Tchernev G, Lotti T. UVA-1 in the treatment of alopecia areata. Dermatol Ther. 2017;30(6):10.1111/dth.12547.

[30]Morsy H, Maher R, Negm D. Correlation between serum IL-17A level and SALT score in patients with alopecia areata before and after NB-UVB therapy. J Cosmet Dermatol. 2018;17(3):533-537.

[31]Perera PY, Lichy JH, Waldmann TA, Perera LP. The role of interleukin-15 in inflammation and immune responses to infection: Implications for its therapeutic use. Microbes Infect. 2012;14:247-61.

[32]Xing L, Dai Z, Jabbari A, Cerise JE, Higgins CA, Gong W, et al. Alopecia areata is driven by cytotoxic $\mathrm{T}$ lymphocytes and is reversed by JAK inhibition. Nat Med. 2014;20:1043-9. 
[33]Marçais A, Cherfils-Vicini J, Viant C, et al. The metabolic checkpoint kinase mTOR is essential for IL-15 signaling during the development and activation of NK cells. Nat Immunol. 2014;15:749-57.

[34]Fuentes-Duculan J, Gulati N, Bonifacio KM, Kunjravia N, Zheng X, Suárez-Fariñas M, Shemer A, Guttman-Yassky E, Krueger JG. Biomarkers of alopecia areata disease activity and response to corticosteroid treatment. Exp Dermatol. 2015.

[35]Fuentes-Duculan J, Gulati N, Bonifacio KM, et al. Biomarkers of alopecia areata disease activity and response to corticosteroid treatment. Exp Dermatol. 2016;25(4):282-286.

[36]Ebrahim AA, Salem RM, El Fallah AA, Younis ET. Serum Interleukin-15 is a Marker of Alopecia Areata Severity. Int J Trichology. 2019;11(1):26-30.

[37]Tabara K, Kozłowska M, Jędrowiak A, Bienias W, Kaszuba A. Serum concentrations of selected proinflammatory cytokines in children with alopecia areata. Postepy Dermatol Alergol. 2019;36(1):63-69.

[38]Bansal A, Relhan V, Garg VK, Saran RK. A cross-sectional study of the histopathology and immunology of alopecia areata: Unearthing the role of the Janus kinase-signal transducer and activator of transcription pathway. Indian J Dermatol Venereol Leprol. 2019;85(5):455461.

[39]Kim JE, Lee YJ, Park HR, Lee DG, Jeong KH, Kang H. The Effect of JAK Inhibitor on the Survival, Anagen Re-Entry, and Hair Follicle Immune Privilege Restoration in Human Dermal Papilla Cells. Int J Mol Sci. 2020;21(14):5137.

[40]Damsky W, King BA. JAK inhibitors in dermatology: The promise of a new drug class. J Am Acad Dermatol. 2017;76(4):736-744.

[41]Almutairi N, Nour TM, Hussain NH. Janus Kinase Inhibitors for the Treatment of Severe Alopecia Areata: An Open-Label Comparative Study. Dermatology. 2019;235(2):130-136. 